'División de Pediatría, Escuela de Medicina, Pontificia Universidad Católica de Chile, Santiago de Chile.

2Unidad de Neurología Pediátrica, División de Pediatría, Escuela de Medicina, Pontificia Universidad Católica de Chile, Santiago de Chile.

${ }^{3}$ Unidad de Genética, División de Pediatría, Escuela de Medicina, Pontificia Universidad Católica de Chile, Santiago de Chile.

Recibido el 5 de marzo de 2015, aceptado el 3 de noviembre de

Correspondencia a:

Dra. Cecilia Mellado

Escuela de Medicina. División de Pediatría, Pontificia Universidad Católica de Chile. Lira 85, Santiago Chile. CP 8330074.

Teléfono: 223548089. Fax: 226384307 cmellado@med.puc.cl

\section{Impacto de la enfermedad genética en los ingresos hospitalarios en un Servicio de Pediatría}

\author{
ANA MOYA ${ }^{1}$, MARTA HERNÁNDEZ ${ }^{2}$, CECILIA MELLADO 3
}

\section{Prevalence of genetic diseases in admissions to a tertiary care hospital pediatric service}

Background: With the epidemiological changes, the role of genetic factors as a cause of morbidity and mortality is increasing, changing disease patterns of patients admitted to pediatric hospitals. Aim: To describe the prevalence of genetic diseases (GD) in patients admitted to a tertiary-care hospital Pediatric Service. Material and Methods: The clinical records of consecutive admissions to a Pediatric Service of a clinical hospital in 2011 were reviewed. Two categories were assigned: with GD and without GD. Both groups were compared according to days of hospitalization, type of admission, readmissions and mortality. Results: We reviewed the $98.1 \%$ of the 1,781 total annual admissions (1,459 cases), 322 of them were readmissions ( 187 cases). The mean age at admission was $54.8 \pm$ 54 months and 55\% were male. The mean hospitalization length was $4.9 \pm 10$ days. Of total admissions and individual cases, 52.7\% (938/1,781) and 48\% (705/1,459) were cases with GD, respectively. Within this group, 85\% (597/705) were sub-categorized as having a significant genetic base. The differences between gender, age average income and hospital mortality were not significant between the two categories. Readmissions were more common for GD than for patients without $G D$ (Odds ratio (OR): 2.6, confidence intervals (CI): 1.9-3.6). Average hospital stay was $27 \%$ higher among GD patients ( $p<0.01$ ). Conclusions: Our findings confirm the high prevalence of GD in pediatric hospitals (52.7\%), with a higher risk for readmission in cases with GD compared with those without GD.

(Rev Med Chile 2016; 144: 188-193)

Key words: Genetic diseases, inborn; Congenital, abnormalities; Pediatric hospitals.

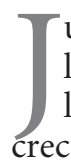
unto con los avances en el conocimiento de la patología genética, la transición epidemiológica y los cuidados de salud, ha habido un creciente rol de los factores genéticos como causa de morbimortalidad en la población pediátrica, constituyendo una causa importante de admisión a los servicios de Pediatría ${ }^{1-4}$. Hall, McCandless y O'Malley han reportado prevalencias de ocupación de camas por niños portadores de alguna enfermedad genética de 53,4\%; 73\% y 50\%, respectivamente $e^{5-7}$.

En Chile, el año 1991, Moreno et al reportaron una prevalencia de enfermedad genética (EG) de 62,5\% en las hospitalizaciones de Cirugía Pediátrica, Neonatología, Unidad de Cuidados Intensivos (UCI) y Pediatría ${ }^{8}$. Desde esa fecha, no hay nuevos reportes sobre prevalencia de EG en servicios hospitalarios en nuestro país.

El objetivo de este estudio fue conocer el impacto de EG en un servicio de Pediatría de alta complejidad y comparar características biodemográficas, rehospitalizaciones y mortalidad con niños hospitalizados sin EG.

El conocimiento de la importancia de la EG en 
pacientes hospitalizados permitiría planificar estrategias asistenciales, formar equipos profesionales multidisciplinarios capacitados en diagnóstico, manejo y tratamiento del paciente y su familia, $y$, a largo plazo, cuantificar costos asociados. Estas estrategias son igualmente necesarias en los cuidados ambulatorios? .

\section{Materiales y Métodos}

El Servicio de Pediatría del Hospital Clínico Pontificia Universidad Católica de Chile (PUC) es un centro de alta complejidad, que recibe pacientes provenientes de todas las regiones del país, servicios de urgencias locales y del Complejo Asistencial Dr. Sótero del Río. El promedio anual de hospitalizaciones en los 3 años previos al estudio fue de 1.974 .

Previa aprobación por el Comité Ético Científico de la Facultad de Medicina de la Pontificia Universidad Católica de Chile, se revisó retrospectivamente la base de datos, epicrisis y fichas clínicas de pacientes menores de 18 años, hospitalizados durante el período de enero a diciembre de 2011. Se excluyeron los casos en los que no se tuvo acceso a las epicrisis, fichas clínicas incompletas y los pacientes hospitalizados en otros servicios pediátricos como neonatología, cirugía y urgencia pediátrica. Se registraron y analizaron las variables sexo, edad de primera hospitalización, motivo de ingreso, diagnósticos de ingreso, días de hospitalización, número de rehospitalizaciones y condición de egreso, y se compararon con las diferentes categorías de enfermedad.

Para este estudio se definió EG como un grupo heterogéneo de afecciones en cuya etiología contribuye significativamente un componente genético $^{10}$. Según esta definición, las admisiones se categorizaron como EG presente o ausente. La categoría con EG se subcategorizó según los criterios del estudio de McCandless ${ }^{6}$ modificado en 3 grupos: base genética significativa, heterogéneas y defectos congénitos de causa desconocida y otros cuadros sindrómicos sin identificación, especificados según la Figura 1. En los casos con diagnósticos complejos, su clasificación se realizó mediante revisión de ficha clínica electrónica y las bases de datos Online Mendelian Inheritance in Man (OMIM), Web Possum, Pubmed y textos de referencia de la especialidad para su adecuada categorización.

Para el análisis estadístico, las variables categóricas se expresaron en número y porcentaje y las variables numéricas en mediana y rango. Para realizar asociación entre variables, se utilizó test de $\chi^{2}$ con valor significativo $\mathrm{p}<0,05$. Para cuantificar asociación se calculó OR con IC de 95\%. Se compararon medias con t de Student. Para análisis de los datos, se usó el programa estadístico SPSS versión 15.

\section{Resultados}

Durante el año 2011 hubo 1.816 hospitalizaciones en el Servicio de Pediatría del Hospital Clínico Pontificia Universidad Católica. Se excluyeron 35 casos en los cuales no se logró obtener información clínica. Para el análisis final se incluyeron 1.781 hospitalizaciones (98,07\% del total), de las cuales 322 fueron readmisiones, correspondiendo a 1.459 casos individuales.

De todas las admisiones, $54,5 \%$ eran de sexo masculino, la mediana de edad fue 34,6 meses (rango intercuartil 10-86 meses). El promedio de días de hospitalización fue 4,85 \pm 10 (Tabla 1 ).

La prevalencia de EG para el total de las hos-

\begin{tabular}{|l|l|}
\hline Categoría & Definiciones \\
\hline $\begin{array}{l}\text { Sin enfermedad } \\
\text { genética }\end{array}$ & $\begin{array}{l}\text { Paciente sano que ingresa por patología aguda. } \\
\text { Enfermedad crónica sin determinante genética aparente. }\end{array}$ \\
\hline $\begin{array}{l}\text { Con enfermedad } \\
\text { genética }\end{array}$ & $\begin{array}{l}\text { Base genética significativa: cromosómicas, monogénicas y multifacto- } \\
\text { riales (anomalías estructurales aisladas o asociadas). } \\
\text { Heterogéneas: patologías con alta sospecha de base genética. } \\
\text { Anomalías congénitas de causa desconocida y otros cuadros sindrómi- } \\
\text { cos sin identificación }\end{array}$ \\
\hline
\end{tabular}

Figura 1. Clasificación de enfermedad genética, según criterio de McCandless modificado. 
Tabla 1. Características de admisiones según categorías de enfermedad, Servicio Pediatría Hospital Clínico Pontificia Universidad Católica, Chile 2011

\begin{tabular}{|c|c|c|c|c|c|c|}
\hline & $\begin{array}{l}\text { Con enfermedad } \\
\text { genética }\end{array}$ & $\begin{array}{l}\text { Sin enfermedad } \\
\text { genética }\end{array}$ & Total & $\begin{array}{c}\text { Valor } \\
\mathbf{p}\end{array}$ & OR & IC \\
\hline Total hospitalizaciones, n (\%) & $938(52,7)$ & $843(47,3)$ & $1.781(100)$ & & & \\
\hline Casos, n (\%) & $705(48,3)$ & $754(51,7)$ & $1.459(100)$ & & & \\
\hline Sexo masculino, n (\%) & $371(53,5)$ & $421(55,5)$ & $792(54,5)$ & 0,10 & & \\
\hline $\begin{array}{l}\text { Promedio edad meses } \\
1{ }^{\circ} \text { hospitalización (DE) }\end{array}$ & $53,4( \pm 55,1)$ & $56,1( \pm 52,7)$ & $54,8( \pm 53,9)$ & 0,38 & & \\
\hline Promedio días hospitalización (DE) & $5,58( \pm 12,3)$ & $4,03( \pm 6,4)$ & $4,9( \pm 10)$ & 0,002 & - & $0,6-2,4$ \\
\hline Hospitalizaciones programadas & $665(82 \%)$ & $146(18 \%)$ & 811 & 0,0001 & 11,6 & $9,3-14,6$ \\
\hline Total rehospitalizaciones & 233 & 89 & 322 & 0,0001 & 2,7 & $2,1-3,6$ \\
\hline Total casos rehospitalizados & 128 & 58 & 186 & 0,0001 & 2,6 & $1,9-3,6$ \\
\hline
\end{tabular}

Tabla 2. Motivo de ingreso y número de rehospitalizaciones, Servicio de Pediatría Hospital Clínico Pontificia Universidad Católica, Chile 2011

\begin{tabular}{|c|c|c|}
\hline & Con EG & Sin EG \\
\hline $\begin{array}{l}\text { Motivo de ingreso } \\
\text { Causa aguda en paciente previamente sano (n, \%) } \\
\text { Causa aguda en paciente crónico con o sin EG (n, \%) } \\
\text { Programada ( } n, \%) \\
\text { Total ingresos }(n, \%)\end{array}$ & $\begin{array}{ll}273 & (29,1) \\
665 & (70,9) \\
938 & (100)\end{array}$ & $\begin{array}{ll}408 & (48,4) \\
289 & (34,3) \\
146 & (17,3) \\
843 & (100)\end{array}$ \\
\hline $\begin{array}{l}\text { Rehospitalizaciones por año } \\
\text { Entre } 2-4(n, \%) \\
\text { Entre } 5-9(n, \%) \\
\text { Más de } 10(n, \%)\end{array}$ & $\begin{array}{rr}117 & (91,4) \\
8 & (6,3) \\
3 & (2,3)\end{array}$ & $\begin{array}{rr}54 & (93,1) \\
3 & (5,2) \\
1 & (1,7)\end{array}$ \\
\hline Total de casos (n, \%) & $128(100)$ & $58(100)$ \\
\hline
\end{tabular}

EG: enfermedad genética.

pitalizaciones fue $52,7 \%(938 / 1.781)$ y para casos individuales $48,3 \%(705 / 1.459)$ (Tabla 1). Para todos los casos individuales anuales, la subcategoría de EG más frecuente fue la de base genética significativa que correspondió a 41\% (597/1.459) de las hospitalizaciones, seguidas de las heterogéneas con 4,5\% (66/1.459) y defectos congénitos de causas desconocidas y otros cuadros sindrómicos sin identificación con 2,9\% (42/1.459); ver Tablas 1 y 3. En el grupo de las EG con base genética significativa, las más frecuentes fueron de etiología multifactorial, seguidas de las monogénicas y las cromosómicas (Tabla 3). Las patologías más frecuentes de esta subcategoría se resumen en la Tabla 4.
Tabla 3. Subcategorías de enfermedad genética en 705 casos hospitalizados, Servicio Pediatría Hospital Clínico Pontificia Universidad Católica, Chile 2011

\begin{tabular}{|lrl|}
\hline Subcategorías & n & $\mathbf{( \% )}$ \\
\hline Base genética significativa & 597 & $(84,7)$ \\
$\quad$ Cromosomopatías & 96 & $(16,1)$ \\
$\quad$ Monogénicas & 99 & $(16,6)$ \\
$\quad$ Multifactorial & 402 & $(67,3)$ \\
Heterogéneas & 66 & $(9,4)$ \\
Anomalías congénitas y otros cuadros & & \\
sindrómicos sin identificación & 42 & $(6,0)$ \\
Total & $705(100)$ \\
\hline
\end{tabular}


Tabla 4. Tipo de EG con base genética significativa hospitalizadas en el Servicio Pediatría Hospital Clínico Pontificia Universidad Católica, Chile 2011

\begin{tabular}{|lcc|}
\hline & n & (\%) \\
\hline Multifactorial & 402 & $(100)$ \\
Cardiopatías congénitas & 294 & $(73,1)$ \\
Malformación SNC & 31 & $(7,7)$ \\
Malformaciones gastrointestinales & 21 & $(5,2)$ \\
Otros & 56 & $(13,9)$ \\
Cromosomopatías & 96 & $(100)$ \\
Síndrome Down & 67 & $(69,8)$ \\
Microdeleción 22q11.2 & 14 & $(14,6)$ \\
Otros & 15 & $(15,6)$ \\
Monogénicas & 99 & $(100)$ \\
Distrofias musculares y miopatías & $23 \quad(23,2)$ \\
Miocardiopatías & 8 & $(8,1)$ \\
Displasias esqueléticas & 7 & $(7,1)$ \\
Síndrome de Wolff-Parkinson White & 6 & $(6,1)$ \\
Otros & $55 \quad(55,5)$ \\
\hline
\end{tabular}

EG: enfermedad genética.

No hubo diferencias significativas en cuanto a sexo ( 53,5 vs $55,5 \%)$, promedio de edad ( 53,4 vs 56,1 meses) (Tabla 1 ) y mortalidad (9 vs 5 casos) para EG vs sin EG (Tabla 5).

En el grupo EG, el promedio días de hospitalización fue de 5,58 vs 4,03 sin EG, valor p 0,001 con IC 0,62-2,48 (Tabla 1).

Al comparar el número de rehospitalizaciones, los individuos con EG se rehospitalizaron más que los individuos sin EG correspondiendo a $72,4 \%$ $(233 / 322)$ vs $27,6 \%(89 / 322)$ del total de las rehospitalizaciones, respectivamente, OR 3,1 (IC: 2,2-4,3) valor $\mathrm{p}<0,005$. Si consideramos los casos individuales, se rehospitalizó 68,8\% (128/186) de los individuos con EG vs $31,2 \%$ de los individuos sin EG, OR 2,1 (IC: 1,5-3,1) (Tabla 1). Algunos casos con EG se rehospitalizaron entre 1-15 veces al año.

En relación al motivo de admisión hospitalaria del total de los ingresos, 47,8\% (851/1.781) correspondieron a ingresos programados y de éstos, $83,7 \%$ (713/851) correspondieron a casos con EG.

\section{Discusión}

Los resultados de este estudio muestran que $52,7 \%$ de los ingresos hospitalarios anuales en un Servicio de Pediatría universitario corresponden a
Tabla 5. Categoría de enfermedad en 14 defunciones hospitalarias, Servicio de Pediatría Hospital Clínico Pontificia Universidad Católica, Chile 2011

\begin{tabular}{lc}
\hline Categorías & n \\
Enfermedad genética & 9 \\
Cardiopatías congénitas & 6 \\
Síndrome Treacher Collins & 1 \\
Síndrome Down y cardiopatía congénita & 1 \\
Linfohistiocitosis hereditaria & 1 \\
Sin enfermedad genética & 5 \\
Leucemia linfoblástica aguda & 3 \\
Hepatoblastoma & 1 \\
Displasia broncopulmonar & 1 \\
Total & 14
\end{tabular}

pacientes con EG. No hubo diferencias en cuanto a distribución por sexo, edad de hospitalización ni mortalidad, entre los individuos con y sin EG durante el año 2011.

Nuestros resultados de frecuencia de EG son similares a 53,4\% y 50\% reportado por Hall en 1978 y O'Malley en el año $2007^{5,7}$, sin embargo, es muy superior a $17 \%, 18,8 \%$ y $21 \%$ reportado por Day en 1973, Páez en 2008 y Lialiaris en $2010^{11-13}$. A su vez, McCandless, el año $2004^{6}$, reportó $73 \%$ y Moreno en 1991, en Chile, $62,5 \%{ }^{8}$, lo que es superior a nuestros hallazgos. Estas diferencias con nuestro trabajo se deben, probablemente, a la población estudiada y a la definición de enfermedad genética de los autores. Creemos que como hospital de referencia y universitario, tenemos un sesgo de selección, ya que se hospitalizan patologías más complejas, a diferencia de Páez y Day, que realizan su trabajo en un hospital de baja complejidad y sin posibilidad de acceder a especialistas en genética.

En cuanto a la definición de enfermedad genética, nosotros excluimos como tales a aquellas con predisposición genética, como asma, epilepsia y retraso del desarrollo psicomotor, las que, por ser un estudio retrospectivo y carecer de información anamnéstica no nos permitió saber si estas eran efectivamente de origen genético o una patología adquirida por patología del parto, infecciones u otros eventos posteriores al nacimiento. La prevalencia del grupo de predisposición genética en nuestro estudio fue de 18,4\% (267 casos), dato no mostrado, si este grupo se hubiera considerado 
en el análisis final dentro de la categoría de EG, esta categoría incrementaría su porcentaje a $71 \%$, similar a $73 \%$ reportado por $\mathrm{McCandless}^{6}$, en donde este grupo correspondió a 37\% del total de las admisiones. Igualmente, el trabajo de Moreno en Chile, del año 1991, incluye predisposición genética, por cuanto la patología más frecuente fueron convulsiones y epilepsia, lo que creemos pudo sobreestimar la prevalencia encontrada.

De la subcategoría de EG con base genética significativa, las más frecuentes fueron las de origen multifactorial $(27,6 \%)$ y de éstas, en nuestra casuística fueron las cardiopatías congénitas con $73,1 \%$, lo que puede ser un sesgo de selección, ya que nuestro hospital es centro médico-quirúrgico cardiológico y con garantía explícita de salud (GES) para cardiopatías congénitas. En este mismo grupo, no pudimos asociar estos casos a una alteración cromosómica, monogénica o a un cuadro sindromático. La mayoría ingresa sólo a resolución quirúrgica de su patología GES, existiendo limitación al acceso y cobertura para la evaluación por especialista en genética y a estudios complementarios.

Las diferencias en el promedio de días de hospitalización entre ambos grupos fue de 5,58 vs 4,03 , lo que alcanza significación estadística con valor $\mathrm{p}=0,01$, pero con IC: $0,62-2,48$. Encontramos dispersión en los días de hospitalización entre individuos portadores de EG vs sin EG, que puede ser explicado porque varias EG requieren de hospitalizaciones cortas para su manejo.

En cuanto al total de rehospitalizaciones, los individuos portadores de EG se rehospitalizan más que los sin EG, siendo esta diferencia estadísticamente significativa, OR 2,1 (IC: 1,5-3,1), similar a lo que reportaron Hall et al, en 1978. En el año $2011,174 / 322$ hospitalizaciones (54\%) correspondieron a EG con base genética significativa multifactorial (102/322) y monogénicas (72/322). Esta subcategoría corresponde a patologías (Tabla 4) que requieren evaluaciones prequirúrgicas, cirugías, tienen más descompensaciones, infecciones recurrentes, medicamentos endovenosos protocolizados que requieren hospitalizaciones más frecuentes.

No se encontró diferencia en la mortalidad intrahospitalaria para las diferentes categorías de enfermedad, que podría explicarse por el bajo número de casos fallecidos en el año de estudio, que correspondió a 0,9\% (14/1.459) de la muestra.

\section{Conclusiones}

La evidencia actual apunta hacia un creciente reconocimiento del papel de los factores genéticos como causa morbimortalidad en todas las edades como efecto de la disminución de la mortalidad infantil, mejores cuidados de salud y mayor sobrevida de pacientes portadores de EG. Este estudio confirma una alta prevalencia de individuos con EG que se hospitalizan, lo que obliga a dirigir nuestros esfuerzos para replantear estrategias asistenciales, formación de recurso humano capacitado, así como en área de la investigación y prevención. Se requieren estudios adicionales al nuestro para conocer las implicaciones futuras e impacto de las enfermedades genéticas en el sistema de salud.

Agradecimientos. A la Dra. Marcela Monge, Jefa de Servicio de Pediatría del Hospital Clínico Pontificia Universidad Católica por su ayuda en facilitar base de datos de las hospitalizaciones del año 2011.

\section{Referencias}

1. Hübner MEG, Nazer JH, De León GJ. [Strategies to improve survival in extreme premature birth]. Rev Chil Pediatr 2009; 80 (6): 551-9.

2. Julio Nazer H, Lucía Cifuentes O. [Congenital malformations in Latin America in the period 1995-2008]. Rev Med Chile 2011; 139 (1): 72-8.

3. Julio NH, Lucía CO. [Prevalence of congenital malformations at birth in Chilean maternity hospitals]. Rev Med Chile 2014; 142 (9): 1150-6.

4. González R, Merialdi M, Lincetto O, Lauer J, Becerra $\mathrm{C}$, Castro R, et al. Reduction in neonatal mortality in Chile between 1990 and 2000. Pediatrics 2006; 117 (5): e949-e954.

5. Hall JG, Powers EK, Mcllvaine RT, Ean VH. The frequency and financial burden of genetic disease in a pediatric hospital. Am J Med Genet 1978; 1 (4): 417-36.

6. McCandless SE, Brunger JW, Cassidy SB. The Burden of Genetic Disease on Inpatient Care in a Children's Hospital. Am J Hum Genet 2004; 74 (1): 121-7.

7. O’Malley M, Hutcheon RG. Genetic Disorders and Congenital Malformations in Pediatric Long-Term Care. J Am Med Dir Assoc 2007; 8 (5): 332-4.

8. Moreno R, Aspillaga M, Avendaño I. [Frequency and characteristics of genetic morbidity in a pediatric hospital]. Rev Chil Pediatr 1991; 62 (2): 112-7.

9. Zubieta-Ruiz B, Sánchez-Márquez P, Castillo-Cruz 
R. Enfermedades genéticas y defectos al nacimiento. Impacto en la morbilidad y mortalidad pediátrica. Acta Pediatr Mex 2009; 30 (4): 220-5.

10. Rimoin DL, Pyeritz RE, Korf B. Emery and Rimoin's Principles and Practice of Medical Genetics. Elsevier B.V. 2013.

11. Lialiaris T, Mantadakis E, Kareli D, Mpountoukas P, Tsalkidis A, Chatzimichail A. Frequency of genetic diseases and health coverage of children requiring ad- mission in a general pediatric clinic of northern Greece. Ital J Pediatr 2010; 36: 9-7288-36-9.

12. Day N, Holmes LB. The incidence of genetic disease in a University hospital population. Am J Hum Genet 1973; 25 (3): 237-46.

13. Páez P, Suárez-Obando F, Zarante I. Enfermedades de origen genético en pacientes pediátricos hospitalizaos en la provincia de Ubate, Colombia. Rev salud pública 2008; 10 (3): 414-22. 